\title{
Anisotropy and inhomogeneity measurement of the transport properties of spark plasma sintered thermoelectric materials
}

\author{
A. Jacquot ${ }^{1}$, M. Rull ${ }^{2}$, A. Moure ${ }^{3}$, J.F. Fernandez-Lozano ${ }^{3}$, M. Martin-Gonzalez ${ }^{2}$, M. Saleemi ${ }^{4}$, \\ M.S. Toprak ${ }^{4}$, M. Muhammed ${ }^{4}$, M. Bartel ${ }^{1}$, M. Jaegle ${ }^{1}$ \\ ${ }^{1}$ Fraunhofer-IPM, Thermoelectric Systems department, Heidenhofstraße 8, 79110 Freiburg, \\ Germany. \\ ${ }^{2}$ Instituto de Microelectrónica de Madrid, C/ Isaac Newton 8. Tres Cantos, 28760 Madrid, Spain. \\ ${ }^{3}$ Instituto de Ceramica y Vidrio, C/ Kelsen, 5 Madrid 28049, Spain \\ ${ }^{4}$ Functional Materials Division, KTH Royal Institute of Technology, Kista-Stockholm, Sweden.
}

\begin{abstract}
The dimensionless figure of merit ZT ranks good thermoelectric materials. Larger electrical conductivity and Seebeck coefficient in conjunction with a low thermal conductivity results in useful thermoelectric materials. Since some of the best thermoelectric materials do have an anisotropic crystal structure and since plasma sintering (SPS) is uniaxial most or all samples produced by SPS should show to some extend anisotropy. In addition, inhomogeneity is also expected in SPS samples because the current density, the temperature field and pressure may not be homogeneous during the sintering process. For the above mentioned reasons, it would be useful to map the transport properties of SPS-samples measured along and perpendicular to the sintering direction. Here we report on the development and capabilities of two new measurement systems developed at Fraunhofer-IPM. The first measurement system is based on an extension of the Van der Pauw method suitable for cube-shaped samples. A mapping of the electrical conductivity tensor of a Skutterudite-SPS samples produced at the Instituto de Microelectrónica de Madrid is presented. The second measurement system is a ZTmeter also developed at the Fraunhofer-IPM. Its enable the simultaneous measurement of the electrical conductivity, Seebeck coefficient and thermal conductivity up to $900 \mathrm{~K}$ of cubes at least $5 \times 5 \times 5 \mathrm{~mm}^{3}$ in size. The capacity of this measurement system for measuring the anisotropy of the transport properties of a $(\mathrm{Bi}, \mathrm{Sb})_{2} \mathrm{Te}_{3}$ SPS sample produced by $\mathrm{KTH}$ is demonstrated by simply rotating the samples.
\end{abstract}

\section{INTRODUCTION}

Thermoelectric materials enter in the fabrication of thermoelectric generators and coolers. The efficiency of those systems is a monotonic growing function of the figure of merit defined as:

$$
Z=\sigma S^{2} / \lambda
$$

Where $\alpha$ is the Seebeck coefficient, $\sigma$ the electrical conductivity and $\lambda$ the thermal conductivity measured all in the same direction. Nevertheless, the thermal conductivity is more conveniently measured along a direction which is perpendicular to the measurement direction of the electrical properties with the measurement systems actually available on the market. This methodology can lead to an overestimation of the figure of merit in the case of a strongly anisotropic crystal structure [1-2]. These overestimated figure-of-merits will never be translated into any improvement of the conversion efficiency in real systems. In addition, lots information about physical phenomenon could be grasped if the anisotropy of the transport properties were more 
easily measured [3-6]. Nevertheless this is not an easy task because the optimal geometry for the electrical properties and thermal conductivity measurements are different for the measurement systems on the market. It has also been noticed that an overestimation of the figure of merit may arise when the samples are inhomogeneous or thermally not stable [7]. Therefore it would be very desirable to measure all the transport properties on the same sample in the same direction.

In this article, we are presenting two solutions for the measurement of the anisotropy of transport properties. The first solution is an extension of the of the Van der Pauw method [8] for cubic samples (3D-VdP). The method presented differs markedly in its simplicity with what have been published up to now [9-15]. This solution is nevertheless restricted to the measurement of the electrical conductivity. The second solution is not restricted to the electrical conductivity and is based on the IPM-ZTmeter [16].

\section{EXPERIMENTAL DETAILS}

\section{D-Van der Pauw method}

The starting material used to test the method was a Skutterudite based wafer provide by Instituto de Microelectrónica de Madrid in spain (wafer ID . CSIC-SPS-641). It was not expected to get any anisotropy of the electrical conductivity because of the crystal structure but the sintering process which is uniaxial can introduce some anisotropy as well as inhomogeneity in the transport properties. The wafer was cut into cubes of size $2 \times 2 \times 2 \mathrm{~mm} 3$ has shown in the insert of the figure 3. Usual VdP measurements have been performed on the face $\mathrm{X}$ and $\mathrm{Y}$ of each carefully oriented cube cubes (figure 1). Resistance measured on the face of the cube $\mathrm{Y}$

along the $\mathrm{X}$ axis was labeled $\boldsymbol{R}_{\boldsymbol{X} \text { Face } \boldsymbol{Y}}$. Assuming an isotropic electrical conductivity perpendicular to the sintering direction (parallel to the Face Y), the resistance $\boldsymbol{R}_{X_{l} \text { Face } \boldsymbol{Y}}$ and $\boldsymbol{R}_{Z_{t} \text { Face } \boldsymbol{Y}}$ must be equal. The results of the measurement in this case is a unique resistance value

\section{$\left(\boldsymbol{R}_{\boldsymbol{X}|| Z_{i} \text { Face } Y}\right)$.}

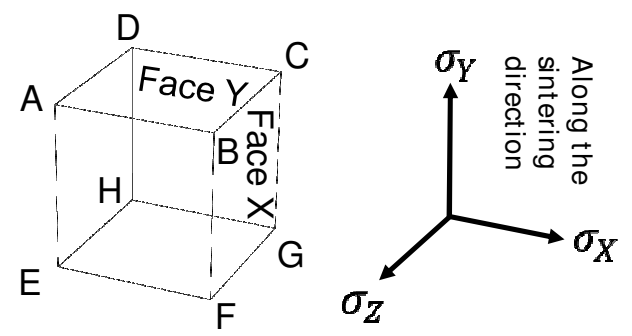

Figure 1. Conventions and labeling used in conjunction with the 3D-VdP method.

When the VdP measurement is performed on the Face $\mathrm{X}$ (or $\mathrm{Z}$ ), two distinct resistance values are obtained $\boldsymbol{R}_{\boldsymbol{X}|| Z_{\text {,Face } \boldsymbol{Y}}} \neq \boldsymbol{R}_{\boldsymbol{X} \mid \boldsymbol{Z}, \text { Face } \boldsymbol{X}}$. In fact it is sufficient to perform the VdP measurement on the face $\mathrm{X}$ (or Z) in order to extract the electrical conductivity along the sintering direction $\left(\boldsymbol{\sigma}_{\boldsymbol{Y}}\right)$ and perpendicular to it $\left(\sigma_{x z}\right)$. This is because the resistance is a function of $\sigma_{Y}, \sigma_{x z}$ and the 
geometry (edge length), the latest being known. $\sigma_{Y}$ and $\sigma_{x z}$ are obtained by reporting the

measured resistance values in the R- $\boldsymbol{\sigma}$ graphs (figure 2) calculated using Comsol Multiphysics [17-18] for the particular geometry and measurement configuration. If a measurement is additionally made on the face $\mathrm{Y}$, it is used only use to confirm the result obtained with the face $\mathrm{X}$ or Z.

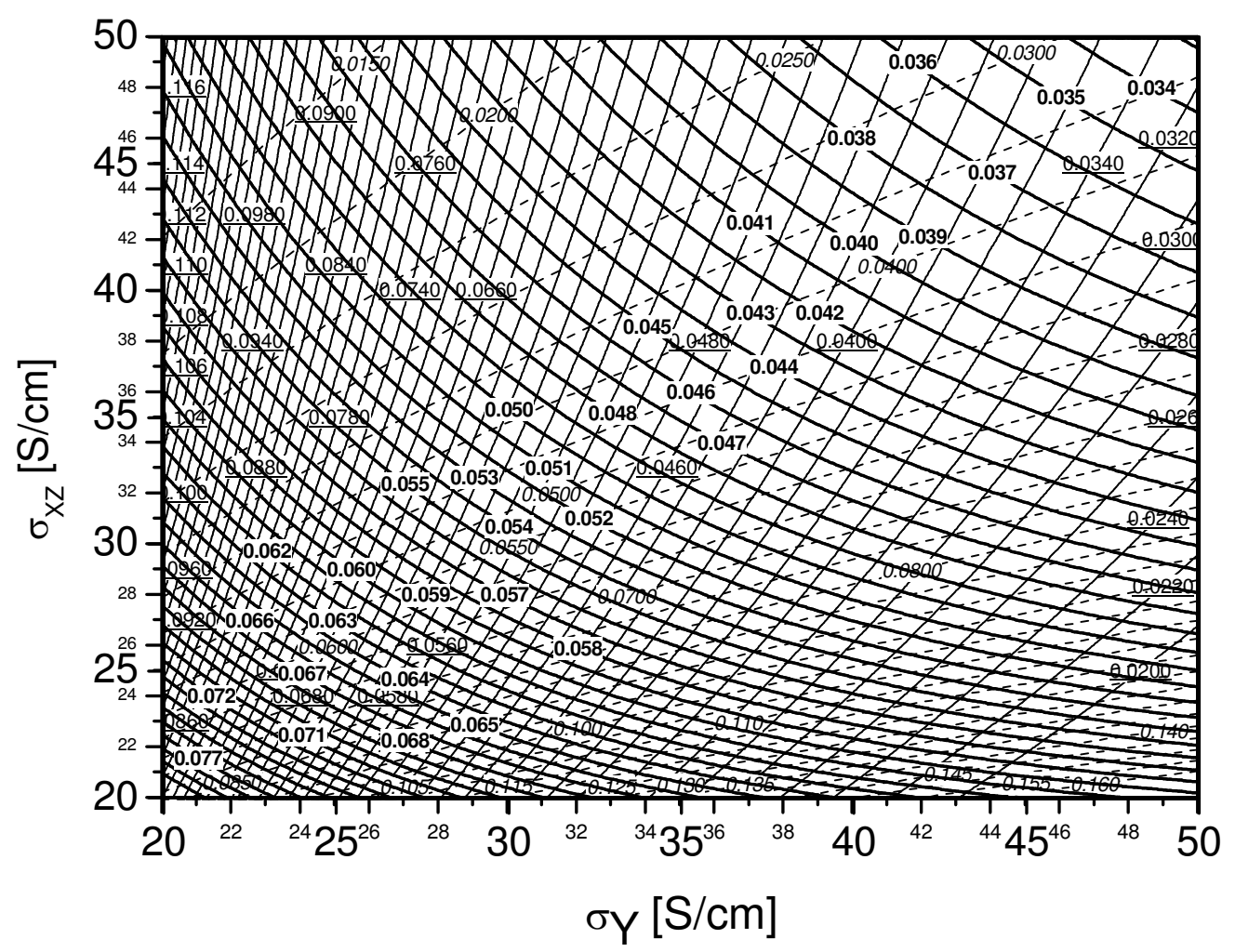

Figure 2. R- $\sigma$ graphs used to extract the electrical conductivity of the sample CSIC-SPS-641 along and perpendicular to the sintering direction. It is the superposition of three R- $\sigma$ diagrams. The labels inside the plot area are resistances calculated for specific faces and direction of a $2 \times 2 \times 2 \mathrm{~mm}^{3}$ sample. The bold solid lines and bold labels represent the contour lines and value of $R_{X \| Z_{Y, F \in Q Y} Y}$, respectively. The thinner solid lines and underlined labels correspond to the contour

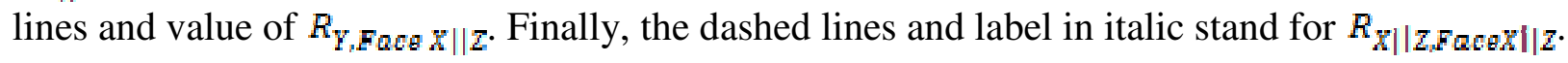



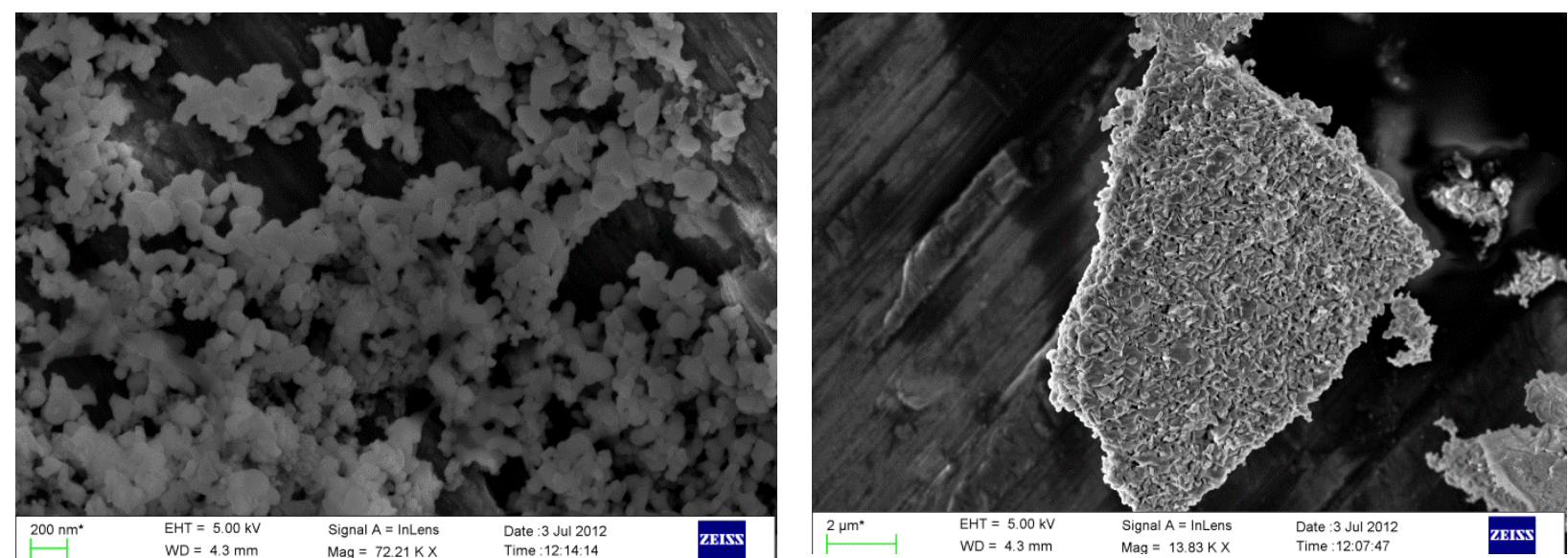

Figure 3. Morphology of the powder that enter in the fabrication of the sample KTH-SPS-16252

\section{IPM-ZTMeter-870K}

All the transport properties were measured in the steady state regime, on the same sample and with a temperature difference along the sample of few Kelvins. The heat flows were measured above and below the sample in order to be able to measure and subtract the heat loss by radiation. The sample measured (KTH_SPS\#16352), 5x5x5 mm $\mathrm{mm}^{3}$ in size, was nanopowder $\mathrm{Bi}_{1.9} \mathrm{Sb}_{0.1} \mathrm{Te}_{3}$ synthetized and sintered at the Royal Institute of Technology in Sweden (KTH). The morphology of the powder is shown in the figure 4. Details about the powder production and compaction can be found in [19]. Morphology of the powder can be show in figure 3.

\section{DISCUSSION}

\section{Mapping of the electrical conductivity and its anisotropy}

Obviously the electrical conductivity of the wafer is inhomogeneous (figure 4). It seems also that the electrically is slightly larger along the sintering direction by few percent.

Nevertheless the anisotropy measured is very small and below the measurement uncertainties or could be attributed to non-identified systematic errors. 


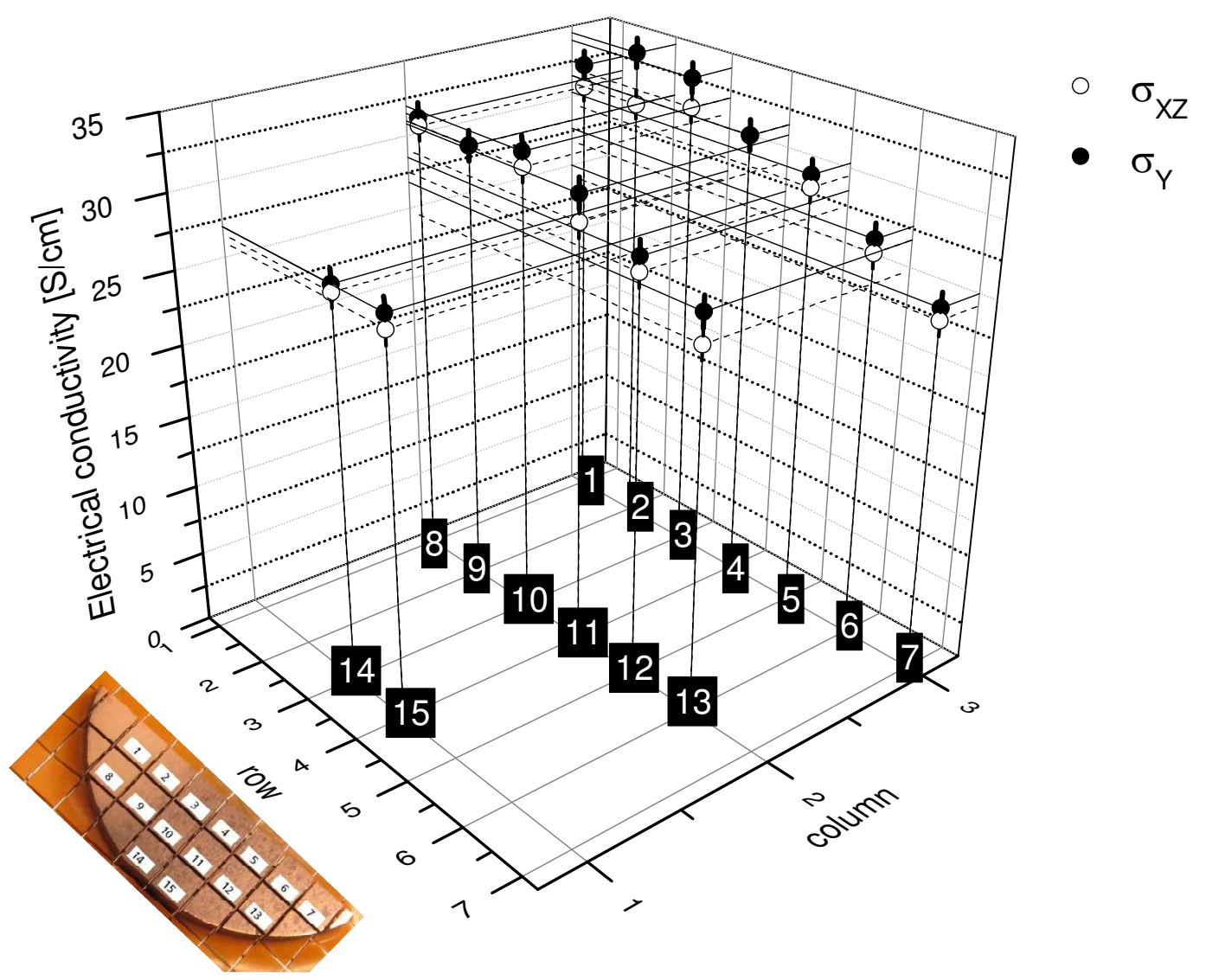

Figure 4. Mapping of the electrical conductivity measured along and perpendicular to the sintering direction of the sample CSIC-SPS-641. The labels with the black background correspond to the labels of the cubes shown in the insert.

\section{Measurement of the anisotropy of the transport properties at high temperatures}

The sample KTH_SPS\#16352 is n-type (figure 5) as expected for this particular chemical composition [20]. N-type soft super lattice based on $\mathrm{Bi}_{2} \mathrm{Te}_{3} /(\mathrm{Bi}, \mathrm{Sb})_{2} \mathrm{Te}_{3}$ created by nanoalloying as also been observed [21]. The anisotropy of the material is very strong even if it was produced from nanopowder. This result is confirmed by a 3D-VdP measurement $\left(\sigma_{X z}=800 \mathrm{~S} / \mathrm{cm} ; \sigma_{Y}=480\right.$ $\mathrm{S} / \mathrm{cm}$ ). The anisotropy of the Seebeck coefficient seems to indicate that there is both electrons and holes in the material, which also tend to explain the rather low ZT measured. 

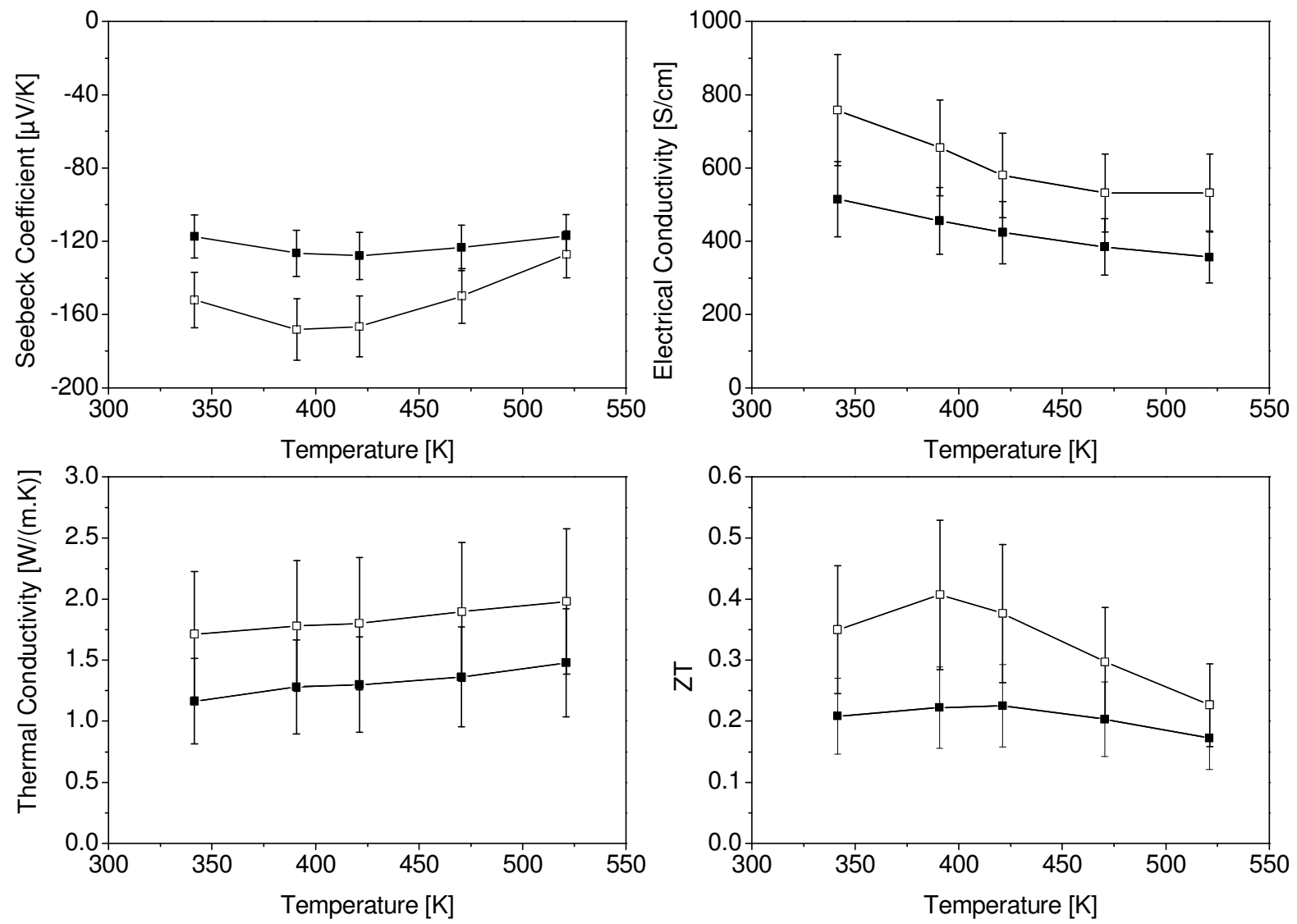

Figure 5: Transport properties measurement of the sample KTH-SPS-16252

\section{CONCLUSIONS}

The 3D-VdP method enables the mapping of the electrical conductivity and its anisotropy The R- $\sigma$ graphs obtained for particular sample geometry frees the end users of having to perform themselves numerical simulations making the method easy to implement. Since it has been proved helpful to us in the purpose of investigating the homogeneity of SPS-samples and to validate the electrical conductivity measurement made with our ZTmeter, this method should find a widespread use. The IPM-ZTmeter has been proved able to measure on a single sample, the anisotropy the transport properties at high temperature using just $5 \times 5 \times 5 \mathrm{~mm}^{3}$ materials. It has been demonstrated that the compaction of nanopowder does not lead systematically to isotropic materials.

\section{ACKNOWLEDGMENTS}

The research work is supported by the European Commission under FP7-NEXTEC project, Grant \# 263167 [FP7/2011-2013]. KTH_SPS\#16352 was produced by Aleksey Ruditskiy who is now working at the City College of New York 


\section{REFERENCES}

[1] A. Jacquot, J. Thomas, J. Schumann, M. Jägle, H. Böttner, T. Gemming, J. Schmidt and D. Ebling, J. Mater. Res. 26, 1773 (2011).

[2] X. Yan, B. Poudel, Y. Ma, W. S. Liu, G. Joshi, H. Wang, Y. Lan, D. Wang, G. Chen and Z. F. Ren, Nano Lett. 10, 3373 (2010).

[3] M. Situmorang and H.G. Goldsmid, Phys. Stat. Sol. (b) 134, K83 (1986).

[4] A. Jacquot, B. Bayer, M. Winkler, H. Böttner, M. Jaegle, J. Solid State Chem. 193, 105 (2012).

[5] A. Jacquot, J. König, B. Bayer, D. Ebling, J. Schmidt, M. Jaegle, Coupled theoretical and experimental investigation of the role of impurity levels and concentration in $\mathrm{Bi}_{2} \mathrm{Te}_{3}$ and $\mathrm{PbTe}$ based materials at high temperature, $8^{\text {th }}$ European Conference on Thermoelectrics, Como, September 22-24, P1-12 (2010).

[6] M. Storder, H.T. Langhammer, phys. Stat. sol (b) 117, 329 (1983).

[7] Von H. Fleischmann, Z. Naturforschg 16 a, 765 (1961).

[8] L.J. van der Pauw, Philips Res. Repts 13, 1 (1958).

[9] D.W. Koon, Rev. Sci. Instrum. 60, 271 (1989).

[10] C. Kasl and M.J.R. Hoch, Rev. Sci. Instrum. 76, 033907 (2005).

[11] I. Kazani, G. De Mey, C. Hertleer, J. Banaszczyk, A. Schwarz, G. Guxho and L. Van Langenhove, Text. Res. J. 0(01) 1 (2011).

[12] J. Kleiza and V. Kleiza, Lith. J. Phys. 45 N5 333 (2005).

[13] Y. Sato and S. Sato, Jpn. J. Appl. Phys. Vol. 40, 4256 (2001).

[14] O. Bierwagen, R. Pomraenke, S. Eilers, and W. T. Masselink, Phys. Rev. B 70, 165307 (2004).

[15] J. de Boor, V. Schmidt, Adv. Mater. 22, 4303 (2010).

[16] A. Jacquot, M. Jaegle, H.-F. Pernau, J. König, K.Tarantik, K. Bartholomé, H. Böttner, "Simultaneous measurement of the thermoelectric properties with the new IPM-ZTMeter", 9th European Conference on Thermoelectrics, Thessaloniki, September 28-30, C-14-P (2011). [17] M. Jaegle, M. Bartel, D. Ebling, A. Jacquot and H. Böttner, "Multiphysics simulation of thermoelectric systems", Proceedings of the $6^{\text {th }}$ European Conference on Thermoelectrics, July 2-4, Paris, 0-27-1 (2008).

[18] D. Ebling, M. Jaegle, M. Bartel, A. Jacquot and H. Böttner, Journal of Electronic Materials 38 Issue 7, 1456 (2009).

[19] M. Saleemi, M. S. Toprak, S. Li, M. Johnsson and M. Muhammed, J. Mater. Chem. 22, 725 (2012).

[20] R. Martin-Lopez, B. Lenoir, A. Dauscher, H. Scherrer and S. Scherrer, Solid State Commun. $108 \mathrm{~N}^{\circ} 5,285$ (1998).

[21] M. Winkler, D. Ebling, H. Böttner, L. Kirste, "Sputtered n-type Soft Super Lattice Thermoelectric Layers Based on $\mathrm{Bi}_{2} \mathrm{Te}_{3} /(\mathrm{Bi}, \mathrm{Sb})_{2} \mathrm{Te}_{3}$ Created by Nanoalloying", Proceedings of the $8^{\text {th }}$ European Conference on Thermoelectrics, September 22-24, Como, 26 (2010). 